GRADIATION\&APPLICATIONS

ISSN 2466-4294 (online) | rad-journal.org

Vol. 2 | Issue 2 | pp. $94-100,2017$

doi: 10.21175/RadJ.2017.02.021

Original research paper

\title{
RADIATION-INDUCED OPTICAL EFFECTS IN CHALCOGENIDE SEMICONDUCTOR GLASSES*
}

\author{
O. Shpotyuk ${ }^{1-3^{* *}}$, M. Shpotyuk4, S. Ubizskii4 \\ IInstitute of Materials of SRC "Carat”, Lviv, Ukraine \\ ${ }^{2}$ Vlokh Institute of Physical Optics, Lviv, Ukraine \\ 3Jan Dlugosz University of Czestochowa, Czestochowa, Poland \\ 4Lviv Polytechnic National University, Lviv, Ukraine
}

\begin{abstract}
A variety of methodological resolutions suitable to identify unambiguously radiation-induced effects in chalcogenide glassy semiconductors is analyzed in details. The radiation-optical effects in chalcogenide glasses are comprehensively considered as resulting from both intrinsic and impurity-related redistribution of covalent chemical bonds known as destruction-polymerization transformations. Two types of experimental measuring protocols are utilized to study the radiation-induced effects within ex-situ direct or in-situ backward chronology, the latter being more adequate for correct testing of competitive inputs from both channels of destruction-polymerization transformations. A critical assessment is given on misleading speculations of some authors ignoring intrinsic radiation-structural transformations in glassy $\mathrm{As}_{2} \mathrm{~S}_{3}$ in view of accompanying oxidation and thermally-induced physical-ageing processes. In final, the $\mathrm{As}_{2} \mathrm{~S}_{3}$ glass is nominated to be the best model object among a wide group of chalcogenide glassy semiconductors revealing the highest sensitivity to radiation-induced metastability.
\end{abstract}

Key words: Chalcogenide glassy semiconductors, optical spectroscopy, $\gamma$-irradiation, metastability, oxidation

\section{INTRODUCTION}

Radiation-induced effects (RIEs) were perfectly justified in chalcogenide glassy semiconductors (ChGSs) since the earliest 1960s, the time of extensive research followed after a pioneer invention of N.A. Goryunova and B.T. Kolomiets on semiconductor properties of these glasses. Afterwards, in the 1970 s and 1980s, the microstructure mechanism of the radiation-induced metastability in different ChGSs became a subject of a scrupulous study in view of many promising applications in radiation dosimetry and radiation-guided modification technologies (see, e.g. ref. [1] and literature therein).

Now, it is commonly accepted that destructionpolymerization transformations (DPTs), i.e. destruction of some covalent chemical bonds in a favor of other ones, followed by structural relaxation towards thermodynamic equilibrium (the phenomenon known as radiation-assisted physical ageing [2]), are principal determinant of RIEs [3-7]. From a purely methodological viewpoint, the RIEs study assumes the development of unprecedented experimental probes to separate contributions arising entirely from radiationinduced DPTs and those caused by the accompanied structural relaxation. The destructed covalent bonds can be renewed in ChGSs intrinsically due to direct interaction of bond-constituting atoms with their neighbors forming a channel for intrinsic bondswitching, or extrinsically due to the interaction with some impurities, thus forming a channel for extrinsic bond-switching. In the former, the pairs of over- and under-coordinated atoms that possess an excess of the positive and negative electrical charge appear $[1,8,9]$. The latter is ascribed to some kinds of impurities formed at the surface of the irradiated ChGSs, the most essential being induced by interaction with oxygen, replacing chalcogen in its bonding configurations within the glass network [1].

Thereby, the intrinsic RIEs in ChGSs are permanently admixed to impurity-related extrinsic ones, forming a complicated scenario of their competitive contribution in an overall balance of detected RIEs. Many of these RIEs are accompanied by relaxation phenomena, such as those typical for multinary bulk ChGSs [10-16] and thin chalcogenide films [17-23].

In realistic conditions, the hierarchical complexity in RIEs reveals at both time-probing and space-holding levels. The former are defined by metastable structural changes detected just after irradiation, which are composed of static (i.e. time-independent or residual) and dynamic (i.e. spontaneously decaying or instable) components, while the second are affected by the locality of the DPTs occurring in sample's bulk [1].

\footnotetext{
* The paper was presented at the Fifth International Conference on Radiation and Applications in Various Fields of Research (RAD 2017), Budva, Montenegro, 2017.

olehshpotyuk@yahoo.com
} 
Under such complicated circumstances, the reliable methodological resolutions allowing unbiased study of RIEs in different ChGS systems have attained a vital importance, especially from practical implementation standpoint.

In this work, at the example of ChGSs of binary arsenic sulfides As-S, including stoichiometric arsenic trisulfide $\mathrm{As}_{2} \mathrm{~S}_{3}$, we give a comparative analysis of different methodological resolutions suitable to unambiguously identify all hierarchical levels in RIEs caused by high-energy $\gamma$-irradiation.

\section{MATERIALS AND METHODS}

2.1. Samples preparation, radiation treatment and optical measurements

The As-S glasses were prepared by conventional melt-quenching route using the respective quantities of high-purity elemental ingredients, as was described in more details elsewhere [7,24,25]. The glasses cut as plane-parallel $1.5 \mathrm{~mm}$ thick plates and carefully polished to high optical quality were annealed before irradiation at temperature $20 \mathrm{~K}$ below the glass transition temperature $\mathrm{T}_{\mathrm{g}}$ to eliminate mechanical stresses frozen within quick cooling.

The $\gamma$-irradiation was carried out at the ambient conditions of a stationary ${ }^{60} \mathrm{Co}$ radiation field (an average energy of $\mathrm{E}=1.25 \mathrm{MeV}$ ). The duration of $\gamma$ treatment (up to 6 months) allows the accumulation of the total dose of $\Phi=2-10 \mathrm{MGy}$. Under such conditions, both intrinsic and impurity-related DPTs are known to be activated [24-27].

Optical transmission spectra of the studied ChGSs were recorded in the region of a fundamental absorption edge using AvaSpec-2048 spectrometer (Avantes, Netherlands) with a maximum error-bar of $\pm 1 \%$. Measurements were performed one month after finishing $\gamma$-irradiation. In such a way, the majority of dynamic decaying changes $[1,28]$ were exhausted, and only static RIEs were detected.

\subsection{Methodology of RIE detection}

Two principally different experimental measuring protocols can be utilized to unambiguously identify the RIEs in ChGSs; these being realized within the continuous (in-situ measurements in direct and/or backward measuring chronology for the same control sample affected by irradiation) or discontinuous (exsitu measurements for control-irradiated sample before and after irradiation, or two samples, one being kept in control-irradiated and other being in referenceunirradiated) cycles (see Fig. 1).

In direct measurements chronology (see Fig. 1a), the optical transmission spectra are recorded ex-situ for the same glass sample taken necessarily in a sequence of the following states:

(1) non-irradiated initial state (i.e. sample taken just before irradiation);

(2) $\gamma$-irradiated state (i.e. sample taken one month after irradiation);

(3) near- $T_{g}$ thermally-annealed state (i.e. deeply aged sample);

95
(4) rejuvenated state (i.e. sample affected to over- $T_{g}$ heating-cooling cycle).

Each of the stages is needed for the chosen ChGS to completely represent the optical signature of the RIEs. To minimize the errors owing to inadequate repetition of too prolonged experimental optical measurements (non-reproducibility of multiple measuring cycles), the glass is positioned in a spectrometer chamber in respect to the probe-light beam, using special marking on its surface. However, the effects of physical ageing in the irradiated ChGS (if any) cannot be resolved adequately within this method.

Therefore, the experimentally measured numerical parameters concerns, in fact, a cumulative input from RIEs and physical ageing.

\begin{tabular}{|c|c|}
\hline \multicolumn{2}{|c|}{$\begin{array}{l}\text { RIE measurements ex-situ } \\
\text { within discontinuous measuring cycle }\end{array}$} \\
\hline $\begin{array}{l}\text { for the same sample: } \\
\text { control (irradiated) }\end{array}$ & $\begin{array}{l}\text { for two samples: } \\
\text { control + reference }\end{array}$ \\
\hline $\begin{array}{c}\text { Chronology: } \\
\text { before and } \\
\text { after irradiation }\end{array}$ & $\begin{array}{l}\text { Chronology: } \\
\text { irradiated vs. }\end{array}$ \\
\hline $\begin{array}{l}\text { Source of false: } \\
\text { (1) non-reproducibility } \\
\text { of measuring cycles } \\
\text { (2) unresolved } \\
\text { natural physical ageing }\end{array}$ & $\begin{array}{l}\text { unirradiated sample } \\
\text { Source of false: } \\
\text { (1) non-reproducibility } \\
\text { of measuring cycles } \\
\text { (2) mismatch between } \\
\text { control-reference samples } \\
\text { (3) unresolved } \\
\text { radiation-assisted ageing }\end{array}$ \\
\hline
\end{tabular}

\begin{tabular}{|c|}
\hline $\begin{array}{c}\text { RIE measurements in-situ } \\
\text { within continuous measuring cycle }\end{array}$ \\
\hline $\begin{array}{c}\text { Chronology: } \\
\text { control (irradiated) } \\
\text { direct }\end{array}$ \\
\hline $\begin{array}{l}\text { Chronology: } \\
\text { backward } \\
\text { (reverse) } \\
\text { (1) sensitivity drift } \\
\text { for too prolonged measuring cycles }\end{array}$ \\
\hline
\end{tabular}

Figure 1. Block-scheme of possible methodological resolutions for ex-situ (a) and in-situ (b) arranged RIE measurements

In principal, the ex-situ measurements in a direct chronology can be realized for two identical ChGS samples taken as the control (i.e. irradiated) and reference (i.e. unirradiated). However, in this case, the samples' identity should be precisely controlled on each stage, as it becomes the main source of false in addition to non-reproducibility of multiple measuring cycles and unresolved radiation-assisted physical ageing (see Fig. 1a).

Within in-situ measurements (see Fig. 1b) in a backward chronology, the optical transmission spectra 
are recorded for the same $\gamma$-irradiated ChGS sample used to reproduce a sequence of the following states:

(1) just irradiated state (i.e. the ChGS sample measured after $\gamma$-irradiation);

(2) annealed state (i.e. the $\gamma$-irradiated sample affected to thermal annealing in a spectrometer chamber);

(3) rejuvenated state (i.e. the thermally-aged $\gamma$ irradiated sample affected to rejuvenation heatingcooling route [2] in spectrometer chamber).

The highest functional reliability can only be provided within this measuring protocol, owing to the complete elimination of the errors associated with (i) the sample reinstallation in a spectrometer chamber and (ii) time separation between subsequent cycles of optical transmission spectra recording for ChGS samples in different states. The contribution of the impurity-related and intrinsic DPTs can be efficiently separated also only within this measuring protocol. Realistically, the in-situ measurements cannot be simply realized in a direct measuring chronology because of difficulties in reliable and highlyreproducible conditions for the unified radiation- and thermally-induced treatment.

\section{RESULTS AND DISCUSSION}

\subsection{RIEs in a direct measuring chronology}

The transmission spectra in the fundamental optical absorption edge of the same stoichiometric $\mathrm{As}_{2} \mathrm{~S}_{3}$ glassy sample measured in a direct chronology, so before (initial thermally-annealed or aged state) and after $\gamma$-irradiation with $\Phi=10.0$ MGy dose and subsequent cycles of thermal annealing at different temperatures are shown in Fig. 2.

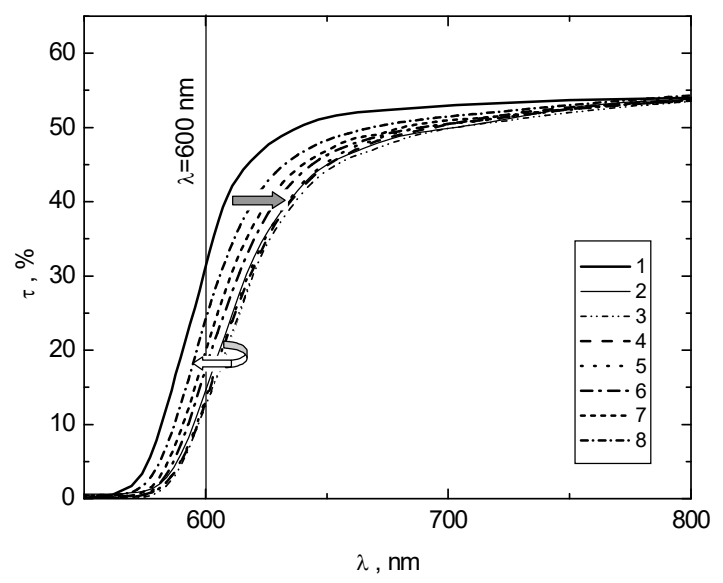

Figure 2. Optical transmission of $\mathrm{As}_{2} \mathrm{~S}_{3}$ glass $(\mathrm{d}=1 \mathrm{~mm})$ measured in direct chronology for initial thermally-annealed (aged) state (1), $\gamma$-irradiated ( $\Phi=10.0 \mathrm{MGy}$ ) state (2) and further thermally aged states due to annealing at 330 (3), 370 (4), 380 (5), 395 (6), 420 (7) and $440 \mathrm{~K}(8)$

The long-wave shift in the optical transmission of the $\gamma$-irradiated ChGS corresponds to the radiation darkening effect [1], which cannot be resolved unambiguously under such ex-situ measuring conditions, as originated from both intrinsic and impurity-related DPTs. Further thermal annealing at growing temperatures, which are close to glass transition temperature $\mathrm{T}_{\mathrm{g}}$, tends optical transmission towards the initial state (with character threshold-like behavior [1]), but this renovation is only partial, because of the competitive contribution from the radiation-induced surface oxidation.

Thus, the initial thermally-aged (curve 1, Fig. 2) and final after-irradiation thermally-aged (curve 8, Fig. 2) states essentially differ in $\mathrm{As}_{2} \mathrm{~S}_{3}$ glass before and after $\gamma$-irradiation, so it is impossible to distinguish between them, unless complete cycle of measurements (curve 1 - curve 2 - curves $3-4-5-6-7-8$, Fig. 2) is performed. The intrinsic and impurity-related DPTs (preferentially due to oxidation) cannot be separated at all in these experiments arranged in a direct measuring chronology. The only resolution is to fully eliminate the oxidized layer from the surface of $\gamma$-irradiated ChGSs and renew the optical measurements for this cleaned sample within the same thermal-treatment cycles.

Fortunately, this resolution is fully applicable for the group of glassy binary arsenic sulfides As-S (incl. stoichiometric $\mathrm{As}_{2} \mathrm{~S}_{3}$ ), since the layer of rhombohedral arsenolite $\mathrm{As}_{4} \mathrm{O}_{6}$, appeared due to the decompositionoxidation reaction activated under normal conditions by high-energy irradiation, can be easily removed from the sample's surface by simple wet cleaning [29-31]. Such arsenolite crystallites act as effective scattering centers, depressing the optical transmission in the near-band-gap region (due to Rayleigh scattering), as well as in the mid-IR optical transmission region (preferentially due to Mie scattering losses) [32]. This specificity is well-illustrated by Fig. 3 showing two principally different positions of the optical transmission edge for uncleaned and cleaned $\gamma^{-}$ irradiated $\mathrm{As}_{2} \mathrm{~S}_{3}$ glass. It is seen that, not only the saturation level, but also the slope of the optical transmission curve are essentially modified in the uncleaned $\gamma$-irradiated stoichiometric $\mathrm{As}_{2} \mathrm{~S}_{3}$ glassy sample affected to surface oxidation.

Noteworthy, this methodological resolution is principally unacceptable for Ge-based ChGSs like glasses of ternary As-Ge-S system [1,3], where the residual effect caused by germanium oxide cannot be simply eliminated due to water insolubility of $\mathrm{GeO}_{\mathrm{x}}$ species [33]. In other words, in RIEs in Ge-based ChGSs studied in direct measuring chronology, we can never distinguish (even roughly) the intrinsic "pure" radiation-induced changes like in binary As-S glasses. So the attempts of some authors [34,35] claiming the Ge-based ChGSs (like glasses of stoichiometric GeS2As2S3 cut-section) as the ideal model object for RIEs are simply wrong and entirely misleading.

Hence, the above direct-chronology measurements have a lot of disadvantages, most of them being connected with time separation and uncertainties between the subsequent cycles of optical transmission spectra recording for initial (thermally-aged or rejuvenated) and final ChGSs (i.e. cleaned or uncleaned after $\gamma$-irradiation). The impossibility to reproduce reliably the exploitation characteristics of the measuring equipment in different conditions is the main obstacle restricting this approach. Simultaneous measurements for different ChGSs samples (one being 
unirradiated and other being $\gamma$-irradiated) allows only partial solution, because of the eventual uncontrolled diversity between samples. Therefore, the optimal testing methodology for intrinsic RIEs is to arrange the measuring cycles for the same ChGS sample, ensuring as small as possible time separation between its different states.

With respect to the optical transmission spectra of stoichiometric $\mathrm{As}_{2} \mathrm{~S}_{3}$ glass measured in a direct chronology for different final states (compare optical transmission spectra shown on Fig. 2 and 3), it is worth to underline that incomplete elimination of the surface oxide layer results only in the partial restoration of initial optical transmission curve. In other words, it means that the $\gamma$-irradiated ChGS sample with some remainders of the oxidation products is always in a more darkened final state as compared with different initial ChGS samples, both thermally-annealed or rejuvenated ones.

This finding is of archetypal importance for the repeatable use of these ChGSs in multiple irradiationannealing measuring cycles (such as those employed in industrial dosimetric systems). Indeed, in this case, the previously $\gamma$-irradiated ChGS samples affected by either thermal annealing or rejuvenation heatingcooling treatment essentially differ from the initial samples not affected by such $\gamma$-irradiation at all (compare optical transmission spectra for initial glassy $\mathrm{As}_{2} \mathrm{~S}_{3}$ on Fig. 2 and two samples shown on Fig. 3).

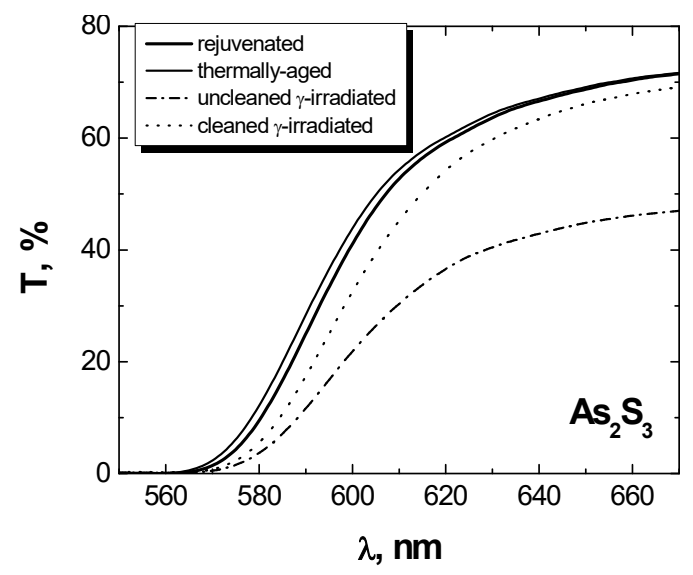

Figure 3. Optical transmission spectra of $\mathrm{As}_{2} \mathrm{~S}_{3}$ glass measured in direct chronology showing two initial states

for rejuvenated (bold curve) and thermally-aged (thin curve) samples, and two final $\gamma$-irradiated states for uncleaned (dot-dashed curve) and cleaned (dotted curve) samples

This approach convincingly discredit some recent speculations [35] which try to ascribe the difference in the optical transmission spectra of two ChGS samples $\left(\mathrm{As}_{2} \mathrm{~S}_{3}\right.$ and $\left.\mathrm{Ge}_{15.8} \mathrm{As}_{21} \mathrm{~S}_{63.2}\right)$, measured a decade after $\gamma$ irradiation ( $\Phi=2.41 \mathrm{MGy}$ ), to intrinsic RIEs. Without comparison for these glasses before irradiation, these data cannot be accepted as a real signature of RIEs. Indeed, in [35], it is ignored that difference in optical transmission spectra could be in these ChGSs just before irradiation, especially if it is accepted that these ChGSs have been affected before to a few subsequent irradiation-annealing cycles (see, for example, the results on $\gamma$-irradiation with doses $\Phi=2.2$ MGy $[3,36,37]$ and $\Phi=1.0$ MGy $[38,39])$.

Most importantly, the shift of optical absorption edge of ChGSs measured in a static position some time after irradiation is a signature of intrinsic RIEs in this glass only in respect to the position of this absorption edge before irradiation.

\subsection{RIEs in a backward measuring chronology}

The breakthrough resolution in RIEs identification is provided by the optical measurements arranged in backward chronology for the same $\gamma$-irradiated ChGS sample. The principal $\gamma$-induced optical effect is considered as consisting of the difference in optical transmission spectra for ChGS sample in $\gamma$-irradiated and subsequently thermally annealed states.

The corresponding measuring protocol is based on the prerequisite that arises intrinsic $\gamma$-induced effect below- $\mathrm{T}_{\mathrm{g}}$ thermal annealing, restoring the initial glass structure [1-3]. In our research, we made one more step to achieve the highest accuracy, performing postirradiation measurements in-situ, i.e. the tested $\gamma$ irradiated ChGS sample was always kept in the same position in respect to the probe-light beam during all cycles of thermal treatments (thermal annealing near $\mathrm{T}_{\mathrm{g}}$ or rejuvenation heating-cooling route).

Thus, this in-situ measuring protocol allows full exclusion of inaccuracies associated with sample's holding in a spectrometer chamber. To distinguish intrinsic and impurity-related RIEs, we also perform our experiments with carefully cleaned ChGS samples free of any products of radiation-induced oxidation (however, in this case, the impurity-related RIEs can be simply decompose from intrinsic RIEs even for uncleaned $\gamma$-irradiated ChGSs).

The optical transmission spectra of the $\gamma$-irradiated (with $\Phi=3$ MGy dose), thermally aged (20 K below $\mathrm{T}_{\mathrm{g}}$ ) and further rejuvenated glassy samples of $\mathrm{As}_{2} \mathrm{~S}_{3}$ and $\mathrm{AsS}_{2}$ compositions recorded in a backward chronology are shown in Fig. 4.

The optical bleaching effect is evident as a result of thermal ageing in both $\gamma$-irradiated samples in full accordance with [2], while rejuvenation occurs an opposite effect. As a result, the optical transmission curves of rejuvenated and $\gamma$-irradiated $\mathrm{AsS}_{2}$ glass coincide, giving an evidence on absence of any intrinsic RIEs in this ChGS. In contrast, the fundamental optical absorption edges in stoichiometric $\mathrm{As}_{2} \mathrm{~S}_{3}$ glass differ essentially in all three states, giving an evidence on comparable intrinsic RIEs and accompanied thermallyassisted physical ageing.

Thus, the high value of RIEs confirms efficiency for both intrinsic and impurity-related DPTs just occurring in stoichiometric $\mathrm{As}_{2} \mathrm{~S}_{3}$ glass under $\gamma$-irradiation. The competitive $\gamma$-induced optical darkening resulting from essential oxidation can be excluded from further consideration by the means of wet surface cleaning, allowing "pure" DPTs caused only by the intrinsic bond redistribution. It means that, in real, the stoichiometric $\mathrm{As}_{2} \mathrm{~S}_{3}$ glass can indeed serve as a model object to study radiation-induced metastability in ChGS systems using different multiexperimental structural probes. 

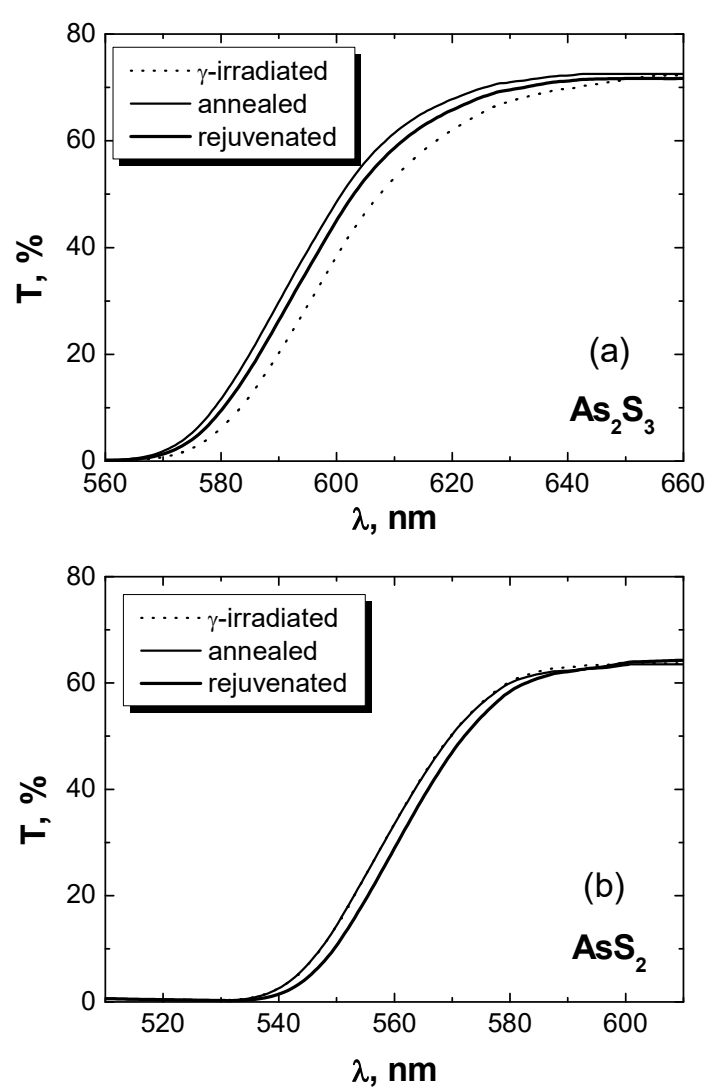

Figure 4. Optical transmission spectra of glassy $\mathrm{As}_{2} \mathrm{~S}_{3}$ (a) and $\mathrm{AsS}_{2}$ (b) samples detected in a backward chronology for $\gamma$-irradiated ( $\Phi=3$ MGy, dotted curve), near- $T_{g}$ annealed (thin curve) and further thermally-rejuvenated (bold curve) states (the sample's surface was preliminary cleaned from products of radiation-induced oxidation).

By finishing, we would like to refer the reader once more to experimentally detected compositional behavior of RIEs in As-S ChGSs measured in-situ in a backward chronology. These samples were irradiated with $0.7 \mathrm{kGy} / \mathrm{h}$ dose rate at near-room temperature (300 K) and total accumulated dose $\Phi=3 \mathrm{MGy}$, as was described in more details elsewhere [24]. After irradiation, these ChGSs were additionally affected to annealing near glass transition temperature $\mathrm{T}_{\mathrm{g}}$. The resulting curve showing difference in optical transmission $\Delta \mathrm{T}_{\max }$ measured in-situ between annealed and $\gamma$-irradiated samples (i.e. detected in a backward measuring chronology) is shown in Fig. 5.

The positive $\Delta \mathrm{T}_{\max }$ value (reaching $\sim 10 \%$ as seen in Fig. 5) corresponds to long-wave shift of fundamental optical absorption edge caused by $\gamma$-irradiation (or, alternatively, to the darkening effect), while the negative $\Delta \mathrm{T}_{\max }$ value (reaching a few \% for S-rich ChGSs) is attributed to opposite short-wave shift (alternatively, to the bleaching effect) caused by postirradiation thermally-assisted physical ageing. Such separation of these contributions (originated from different effects (intrinsic radiation transformations, radiation-induced oxidation, ageing and thermallyassisted ageing) is possible only for measurements arranged in-situ in a backward chronology.

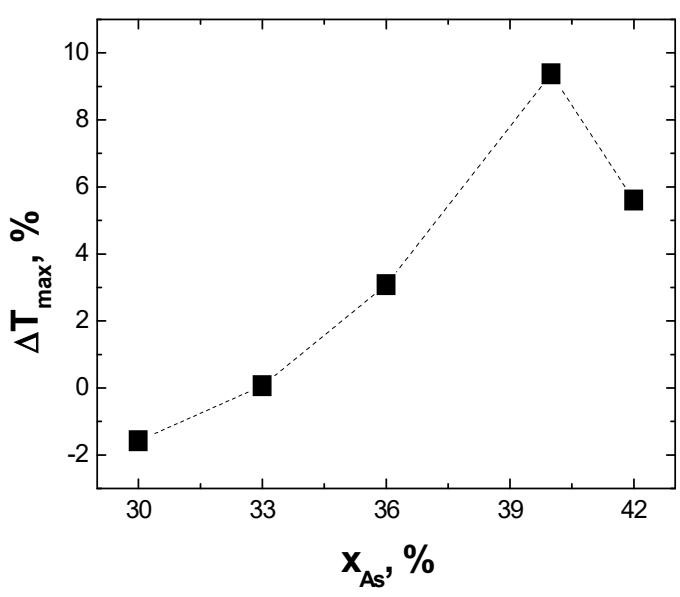

Figure 5. Compositional behavior of the RIEs ( $\Phi=\sim 3$ MGy) in As-S glasses exemplified by changes in optical transmittance due to long-wavelength shift of fundamental absorption edge detected in-situ in a backward measuring chronology respectively to thermally-annealed samples.

\section{CONCLUSIONS}

The methodological resolutions suitable to identify unambiguously radiation-induced optical effects in chalcogenide glasses are systematically analyzed and critically compared with alternative approaches. The studied radiation-optical effects are comprehensively considered as resulting from both intrinsic and impurity-related redistribution of covalent chemical bonds in chalcogenide glasses known as destructionpolymerization transformations.

Two types of experimental measuring protocols can be utilized to study the radiation-induced effects within ex-situ direct or in-situ backward chronology, the latter being more adequate for correct testing of competitive inputs arising from the intrinsic and impurity-related transformations, as well as accompanied physical ageing effects. A critical assessment is given on the misleading speculations of some authors ignoring intrinsic radiation-induced structural transformations in glassy $\mathrm{As}_{2} \mathrm{~S}_{3}$ in view of accompanying processes of radiation-induced oxidation and thermally-induced physical ageing. In final, the stoichiometric $\mathrm{As}_{2} \mathrm{~S}_{3}$ glass is nominated to be the best model object among a wide group of chalcogenide glasses revealing the highest sensitivity to radiation-induced metastability.

Acknowledgement: The paper is a part of research done within the projects No 0116 Uoo2578 (subject of Scientific Program funded by Ministry of Education and Science of Ukraine for years 2016-2018). MSh acknowledges financial support from Ministry of Education and Science of Ukraine within the funding program DB/Nanosensor (No O116Uoo4411).

\section{REFERENCES}

1. O. I. Shpotyuk, "Radiation-induced effects in chalcogenide vitreous semiconductors," in Semiconducting Chalcogenide Glass I. Glass 
O. Shpotyuk et al., Radiation-induced optical effects..., Rad. Applic., 2017, 2, 2, 94-100

Formation, Structure, and Stimulated Transformations in Chalcogenide Glasses, R. Fairman, B. Ushkov, Eds., Cambridge (MA), USA: Elsevier Academic Press, 2004, ch. 6, pp. 215 - 260.

DOI: 10.1016/Soo80-8784(04)80048-6

2. O. Shpotyuk, R. Golovchak, A. Kozdras, "Physical ageing of chalcogenide Glasses," in Chalcogenide Glasses: Preparation, Properties and Applications, J. L. Adam, X. Zhang, Eds., Sawston, UK: Woodhead Publishing, 2013, ch. 13, pp. $209-264$ DOI: $10.1533 / 9780857093561.1 .209$

3. O. I. Shpotyuk et al., "Compositional trends in radiation-optical properties of chalcogenide glasses," J. Optoelectron. Adv. Mater., vol. 4, no. 3, pp. 751 762, Sep. 2002

Retrieved from:

http://citeseerx.ist.psu.edu/viewdoc/download?doi=10. 1.1.614.930\&rep=rep1\&type $=$ pdf Retrieved on: Jan. 15, 2017

4. O. I. Shpotyuk, "Induced effects in chalcogenide glassy semiconductors and destruction-polymerization transformations concept," Latv. J. Phys. Techn. Sci., vol. 4, pp. $32-43,1993$.

5. O. Shpotyuk et al., "Destruction-polymerization transformations as a source of radiation-induced extended defects in chalcogenide glassy semiconductors," Phys. Stat. Sol. C, vol. 10, no. 1, pp. $125-128$, Jan. 2013. DOI: $10.1002 /$ pssc.201200407

6. Ya. Shpotyuk et al., "Radiation-induced effects in chalcogenide amorphous semiconductors: On the role of destruction-polymerization transformations," J. Non-Crystr. Sol., vol. 377, pp. 46 - 48, Oct. 2013. DOI: 10.1016/j.jnoncrysol.2013.01.054

7. M. V. Shpotyuk et al., "On the origin of radiationinduced metastability in vitreous chalcogenide semiconductors: The role of intrinsic and impurityrelated destruction-polymerization transformations," Semicond. Phys. Quant. Electron. Optoelectron., vol. 18, no. 1, pp. 90 - 96, Feb. 2015.

DOI: $10.15407 /$ spqe018.01.090

8. O. I. Shpotyuk, "Mechanism of radiation-structural transformations in amorphous $\mathrm{As}_{2} \mathrm{~S}_{3}$," Rad. Effects and Defects in Solids, vol. 132, no. 4, pp. 393 - 396, 1994. DOI: $10.1080 / 10420159408219992$

9. O. I. Shpotyuk et al., "Radiation-stimulated processes in vitreous arsenic trisulphide," J. Non-Cryst. Solids, vol. 176, no. 1, pp. 45 - 50, Oct. 1994. DOI: 10.1016/0022-3093(94)90209-7

10. D. Zhao et al., "Gamma-ray-induced multi-effect on properties of chalcogenide glasses," J. Am. Ceram. Soc., vol. 89, no. 11, pp. $3582-3584$, Nov. 2006 DOI: $10.1111 / \mathrm{j} .1551-2916.2006 .01268 . x$

11. F. Xia et al., "Gamma ray irradiation induced optical band gap variations in chalcogenide glasses," Nucl. Instr. Meth. Phys. Res. B, vol. 234, no. 4, pp. 525 - 532, Jul. 2005.

DOI: 10.1016/j.nimb.2005.02.019

12. F. Xia et al., " $\gamma$-ray irradiation induced multiple effects on Ge-as-Sde chalcogenide glasses," $J$. Non-Cryst. Solids, vol. 354, no. 12-13, pp. 1365 - 1368, Feb. 2008. DOI: 10.1016/j.jnoncrystsol.2006.11.049

13. W. Shen et al., "Gamma-ray irradiation resistance of silver doped GeS2-Ga2S3-AgI chalcogenide glasses," Nucl. Instr. Meth. Phys. Res. B, vol. 329, pp. $48-51$, Jun. 2014.

DOI: 10.1016/j.nimb.2014.02.119

14. W. Shen et al., "Controllable gamma-ray sensitivity of Ag-doped and/or AgI-modified Ge-Ga-S glasses," Nucl. Instr. Meth. Phys. Res. B, vol. 280, pp. $36-38$, Jun. 2012.

DOI: 10.1016/j.nimb.2012.02.034
15. O. A. Lafi et al., "The effect of gamma irradiation on glass transition temperature and thermal stability of $\mathrm{Se}_{96} \mathrm{Sn}_{4}$ chalcogenide glass," Rad. Phys. Chem., vol. 79, no. 1, pp. 104 - 108, Jan. 2010. DOI: 10.1016/j.radphyschem.2009.08.005

16. M. A. Al-Ewaisi et al., "Effect of gamma irradiation on some electrical properties and optical band gap of bulk $\mathrm{Se}_{92} \mathrm{Sn}_{8}$ chalcogenide glass," Physica B, vol. 405 , no. 12, pp. $2643-2647$, Jun. 2010.

DOI: 10.1016/j.physb.2010.03.045

17. S. K. Troipathi, "Irradiation induced changes in semiconducting thin films," Defects Diffusion Forum, vol. 341, pp. 181 - 219, Jul. 2013. DOI: 10.4028/www.scientific.net/DDF.341.181

18. S. M. El-Sayed, "Electron beam and gamma irradiation effects on amorphous chalcogenide SbSe2.5 films," Nucl. Instr. Meth. Phys. Res. B, vol. 225, no. 4, pp. 535 - 543, Oct. 2004. DOI: 10.1016/j.nimb.2004.05.033

19. D. Sarkar et al., "Influence of electron beam irradiation on structural and optical properties of thermally evaporated GeTe thin films," Rad. Phys. Chem., vol. 98, pp. $64-68$, May 2014. DOI: 10.1016/j.radphyschem.2014.01.016

20. M. R. Balboul, "Optical effects induced by gamma and UV irradiation in chalcogenide glass," Rad. Measurements, vol. 43, no. 8, pp. 1360 - 1364 Sep. 2008. DOI: 10.1016/j.radmeas.2008.03.007

21. M. R. Balboul et al., "Effect of $\mathrm{Co}^{60} \gamma$-irradiation on the optical properties of thin films from the system $\mathrm{GeSe}_{3}-\mathrm{Sb}_{2} \mathrm{Se}_{3}-\mathrm{ZnSe}$," Rad. Phys. Chem., vol. 81, no. 12, pp. $1848-1855$, Dec. 2012. DOI: 10.1016/j.radphyschem.2012.08.007

22. G. A.N. Amin et al., "Optical response of gamma irradiated arsenic selenide thin films," Mater. Sci. Semicond. Processing, vol. 15, no. 4, pp. 455-459, Aug. 2012. DOI: 10.1016/j.mssp.2012.03.017

23. G. A. N. Amin et al., "Study of gamma-radiationinduced optical effects in Ge-Se-Cd for possible industrial dosimetric applications," Rad. Phys. Chem., vol. 72, no. 4, pp. 419 - 422, Mar. 2005. DOI: $10.1016 / \mathrm{j}$. radphyschem.2004.03.013

24. M. Shpotyuk et al., "Compositional trends of $\gamma$-induced optical changes observed in chalcogenide glasses of binary As-S system,” J. Non-Cryst. Solids, vol. 386, pp. 95 - 99, Feb. 2014. DOI: 10.1016/j.jnoncrysol.2013.12.001

25. O. Shpotyuk et al., "Structural-relaxation phenomena in As-S glasses as probed by combuned PAL/DBAR technique," Mat. Chem. Phys., vol. 155, pp. 76-82, Apr. 2015 DOI: 10.1016/j.matchemphys.2015.02.001

26. M. Shpotyuk et al., "FSDP-related correlations in $\gamma$-irradiated chalcogenide semiconductor glasses: The case of glassy arsenic trisulphide g- $\mathrm{As}_{2} \mathrm{~S}_{3}$ revised," J. Phys. Chem. Sol., vol. 74, no. 12, pp. $1721-1725$, Dec. 2013. DOI: 10.1016/j.jpcs.2013.06.016

27. M. Shpotyuk et al., "Surface oxidation in glassy arsenic trisulphide induced by high-energy $\gamma$-irradiation," Rad. Phys. Chem., vol. 97, pp. 341 - 345, Apr. 2014. DOI: 10.1016/j.radphyschem.2013.12.021

28. V. Balitska et al., "Post-irradiation relaxation in vitreous arsenic/antimony trisulphides," J. Non-Cryst. Solids, vol. 357, no. 2, pp. 487- 489, Jan. 2011. DOI: $10.1016 /$ j.jnoncrysol.2010.06.052

29. J. S. Berkes et al., "Photodecomposition of amorphous $\mathrm{As}_{2} \mathrm{Se}_{3}$ and $\mathrm{As}_{2} \mathrm{~S}_{3}$," J. Appl. Phys., vol. 42, pp. $4908-$ 4916, 1971.

DOI: 10.1063/1.1659873 
30. S. A. Keneman et al., "Evaporated films of arsenic trisulfide: Physical model of effects of light exposure and heat cycling," J. Appl. Phys., vol. 49, pp. $4663-$ 4673, 1978.

DOI: $10.1063 / 1.325555$

31. M. Shpotyuk et al., "Surface oxidation in glassy arsenic trisulphide induced by high-energy $\gamma$-irradiation," Rad. Phys. Chem., vol. 97, pp. 341 - 345, Apr. 2014. DOI: $10.1016 /$ j.radphyschem.2013.12.021

32. J. S. Sanghera et al., "Effect of scattering centers on the optical loss of $\mathrm{As}_{2} \mathrm{~S}_{3}$ glass fibers in the infrared," $J$. Appl. Phys., vol. 75, no. 10, pp. 4885 - 4891, Jan. 1994. DOI: $10.1063 / 1.355774$

33. S. Lovas et al., "The room temperature visible photoluminescence in $\mathrm{g}-\mathrm{As}_{2} \mathrm{~S}_{3}$ and Ge-based glasses," Sci. Bull. Uzhgorod Univ. Ser. Fiz., vol. 34, pp. $54-58$, 2013.

Retrieved from:

http://dspace.uzhnu.edu.ua/jspui/handle/lib/2625

Retrieved on: Jan. 10, 2017

34. T. S. Kavetskyy and A. L. Stepanov, "Effects of gammairradiation and ion implantation in chalcogenide glasses," in Glass Nanocomposites: Synthesis, Properties and Applications, B. Karmakar, K. Rademann, A. L. Stepanov, Eds., Cambridge (MA), USA: Elsevier Acad. Press, 2016, ch. 14, pp. $341-358$

DOI: 10.1016/B978-0-323-39309-6.00014-6
35. T. S. Kavetskyy, "Long-term radiation-induced optical darkening effects in chalcogenide glasses," Semicond. Phys. Quantum Electron. Optoelectron., vol. 19, no. 4, pp. $395-398,2016$.

DOI: $10.15407 /$ spqeo19.04.395

36. O. I. Shpotyuk et al., "Radiation optical effects in As2S3-GeS2 semiconducting glasses," Phys. Chem. Glasses, vol. 42, no. 2, pp. 95-98, Apr. 2001.

37. E. Skordeva et al., " $\gamma$-induced changes in Ge-As-S glasses,” J. Optoelectron. Adv. Mater., vol. 2, pp. 259 266, 2000.

Retrieved from:

https://www.researchgate.net/publication/242238293 G-Induced Changes in Ge-As-S Glasses Retrieved on: Jan. 10, 2017

38. V. Balitska et al., "On the instability effects in radiationsensitive chalcogenide glasses," Rad. Measurements, vol. 42, no. 4-5, pp. 941 - 943, Apr-May 2007. DOI: 10.1016/j.radmeas.2007.02.045

39. V. Balitska et al., "Dynamic radiation-induced effects in chalcogenide vitreous compounds," J. Non-Cryst. Solids, vol. 287, no. 1-3, pp. 216 - 221, Jul. 2001.

DOI: 10.1016/Soo22-3093(01)00635-4 